\title{
Asthma in Patients With Japanese Cedar Pollinosis
}

\author{
Akihiko Tanaka, MD, PhD, ${ }^{1}$ Kenji Minoguchi, $M D, P h D,{ }^{2}$ Ruby Pawankar, $M D, P h D,{ }^{3}$ \\ and Mitsuru Adachi, $M D, P h D^{1}$
}

\begin{abstract}
Japanese cedar pollen is the most common causative allergen for seasonal allergic rhinitis (AR) in Japan. More commonly known as Japanese cedar pollinosis, it occurs in spring causing the typical symptoms of seasonal AR, such as sneezing, rhinorrhea, nasal obstruction, nasal itching, and itching of the eyes. Previous reports indicate that the prevalence of Japanese cedar pollinosis among Japanese was $26.5 \%$. According to a more recent questionnaire-based survey, the prevalence of Japanese cedar pollinosis in patients with adult asthma might be up to $30 \%$ to $50 \%$, suggesting higher rates than that previously reported. Moreover, $30 \%$ to $60 \%$ of adult asthmatic patients with concomitant pollinosis have exacerbations of their asthma symptoms during the Japanese cedar pollen season. These findings suggest that concomitant Japanese cedar pollinosis may be an aggravating factor in patients with asthma. As with other pollens, such as grass and birch, Japanese cedar pollen was shown to be a trigger factor for worsening asthma. In clinical practice, a number of Japanese patients with asthma are monosensitized to Japanese cedar pollen but not to other antigens. Further studies are needed to elucidate the mechanisms of Japanese cedar pollen in inducing and in exacerbating asthma. The presence of concomitant $\mathrm{AR}$ is often associated with the difficulty in asthma control. However, there has been a controversy whether treating concomitant AR by intranasal corticosteroid would produce better asthma-related outcomes in patients with asthma and AR. The effect of treating concomitant cedar pollinosis by intranasal corticosteroids on asthma control in patients with asthma and cedar pollinosis also remains unknown. Certain systemic treatments, such as leukotriene receptor antagonist and anti-IgE monoclonal antibody, are supposed to reduce the symptoms of both asthma and AR in patients with asthma and concomitant AR. In conclusion, Japanese cedar pollinosis is often associated with exacerbations of asthma. Further investigations are expected to elucidate the precise impact and mechanisms of Japanese cedar pollinosis in asthma.
\end{abstract}

Key Words: Japanese cedar pollinosis, asthma, allergic rhinitis

(WAO Journal 2012; 5:S218-S222)

From the ${ }^{1}$ Division of Allergy and Respiratory Medicine, Department of Internal Medicine, Showa University, Tokyo, Japan; ${ }^{2}$ Sumiregaoka Clinic, Kanagawa, Japan; ${ }^{3}$ Nippon Medical School, Tokyo, Japan.

Presented at the World Allergy Congress 2007 in Thailand.

Supported by educational grant from BANYU Co.

The authors have no funding or conflicts of interest to disclose.

Correspondence to: Akihiko Tanaka, MD, PhD, Division of Allergy and Respiratory Medicine, Department of Internal Medicine, Showa University, School of Medicine, 1-5-8 Hatanodai, Shinagawa-ku, Tokyo 142-8666, Japan. Telephone: +81-3-3784-8532. Fax: +81-3-37848742. E-mail: tanakaa@med.showa-u.ac.jp.

Copyright (c) 2012 by World Allergy Organization

\section{ASTHMA AND JAPANESE POLLINOSIS}

apanese cedar pollen is the most common allergen, which elicits seasonal allergic rhinitis (AR) in spring, in Japan. An alarming number of the patients with Japanese cedar pollinosis suffer from symptoms, such as rhinorrhea, nasal obstruction, nasal itching, sneezing, and itching of the eyes. According to the survey based on a questionnaire, the prevalence of AR and Japanese cedar pollinosis was $23.4 \%$ and $26.5 \%$, respectively, in Japan. ${ }^{1}$ In recent years, Japanese cedar pollinosis is called as "a folk disease" in Japan, due to the increase in the number of patients and increase of the related medical costs. In addition, lowering trend in the age of patients with Japanese cedar pollinosis has been noted in recent years. Prevalence rate of Japanese cedar pollinosis in children was reported to be $13.8 \%$ to $22.9 \%{ }^{2}$ This survey was executed in 407 to 510 children in May or June every year from 1995 to 2001. Prevalence rate of pollinosis was found to be related to the amount of pollen in the air. The amount of pollen in the air differs by the area and the year. Okamoto ${ }^{3}$ reported that $60 \%$ of elementary school students in Yamanashi prefecture, who had been exposed to high amounts of pollen, showed high levels of specific IgE antibodies to Japanese cedar pollen in sera.

$\mathrm{AR}$ and asthma frequently coexist. In previous reports, $30 \%$ to $40 \%$ of the patients with AR were found to have concomitant asthma, and $50 \%$ to $80 \%$ of the patients with asthma had concomitant AR. ${ }^{4}$ A large-scale epidemiological survey in Tohoku area was reported in 2009 by Yamauchi et al. ${ }^{5}$ In this survey, 6064 patients with asthma and 3945 patients with AR were enrolled. Of 2781 patients with adult asthma, 1693 (60.8\%) patients had AR symptoms, and 1155 (41.5\% in 2781 ) patients were eventually diagnosed with AR. Likewise, among 3283 patients with childhood asthma, 1335 (40.6\%) patients were eventually diagnosed with AR. Meanwhile, $49.0 \%$ of 3945 patients with AR showed asthmatic symptoms and $34.8 \%$ (in 3945) were eventually diagnosed with asthma, suggesting the high prevalence of asthma in patients with AR.

Prevalence of AR in atopic asthmatic patients is higher than that in nonasthmatic patients. Likewise, in clinical practice, the patients with asthma are often associated with Japanese cedar pollinosis; in addition, the patients with asthma and concomitant Japanese cedar pollinosis often show exacerbation in the season of Japanese cedar pollen. Therefore, we investigated the prevalence of concomitant asthma and Japanese cedar pollinosis, and the impact of Japanese cedar pollinosis on asthmatic control in 333 patients with adult asthma. ${ }^{5}$ Overall, 333 asthmatic patients, whose mean age was $48.3 \pm 1.0$ years and ranged from 15 to 83 years, 
TABLE 1. Patient Characteristics

\begin{tabular}{|c|c|c|c|}
\hline & Total & Cedar Pollinosis $(-)$ & Cedar Pollinosis $(+)$ \\
\hline No. patients & 333 & 217 & 116 \\
\hline Age, yr & $48.3 \pm 1.0$ & $48.7 \pm 1.3$ & $47.5 \pm 1.6$ \\
\hline \multicolumn{4}{|l|}{ Sex, n (\%) } \\
\hline Male & $137(41.1)$ & $91(41.9)$ & $46(39.7)$ \\
\hline Female & $196(58.9)$ & $126(58.1)$ & $70(60.3)$ \\
\hline Type Atopic, n (\%) & $211(63.4)$ & $129(59.5)$ & $82(70.7)$ \\
\hline Nonatopic & $122(36.6)$ & $88(40.5)$ & $34(29.3)$ \\
\hline Duration of asthma, yr & $15.3 \pm 0.8$ & $15.0 \pm 1.0$ & $15.8 \pm 1.3$ \\
\hline IgE RIST (U/mL) & $565.8 \pm 51.6$ & $580.6 \pm 72.9$ & $538.9 \pm 60.3$ \\
\hline Cedar RAST (UA/mL) & $10.9 \pm 1.4$ & $4.2 \pm 1.2$ & $23.4 \pm 2.8$ \\
\hline Histamine- $\mathrm{PC}_{20}(\mu \mathrm{g} / \mathrm{mL})$ & $1424 \pm 194(\mathrm{n}=125)$ & $1550 \pm 262(\mathrm{n}=81)$ & $1193 \pm 267(n=44)$ \\
\hline \multicolumn{4}{|l|}{ Severity of asthma, n (\%) } \\
\hline Mild & $81(24.3)$ & $54(24.9)$ & $27(23.3)$ \\
\hline Moderate & $177(53.2)$ & $116(53.5)$ & $61(52.6)$ \\
\hline Severe & $75(22.5)$ & $47(21.6)$ & $28(24.1)$ \\
\hline \multicolumn{4}{|l|}{ Therapy, n (\%) } \\
\hline BDP & $275(82.6)$ & $182(83.9)$ & $93(80.2)$ \\
\hline Slow-release theophylline & $259(77.8)$ & $165(76.0)$ & $94(81.0)$ \\
\hline Oral $\beta 2$-agonists & $126(37.8)$ & $77(35.5)$ & $49(42.2)$ \\
\hline Anti-allergic agents, n (\%) & $57(17.1)$ & $37(17.1)$ & $20(17.2)$ \\
\hline Oral steroids, n (\%) & $20(6.0)$ & $13(6.0)$ & $7(6.0)$ \\
\hline
\end{tabular}

Adapted from Ueno et al (2002) with permission from Arerugi. ${ }^{6}$

RIST, Radio ImmunoSorbent Test; RAST, Radio AllergoSorbent Test.

were enrolled in the study (137 males and 196 females). The prevalence of Japanese cedar pollinosis was 34.8\% (116 patients). Patients' characteristics are shown in Table 1. Distribution of the population classified by severity was similar between the groups with and without cedar pollinosis (Table 1). Asthmatic patients with Japanese cedar pollinosis were found across all age groups (Fig. 1). Among 116 asthmatic patients with Japanese cedar pollinosis, 41 (35.3\%) patients reported that their asthmatic control was aggravated

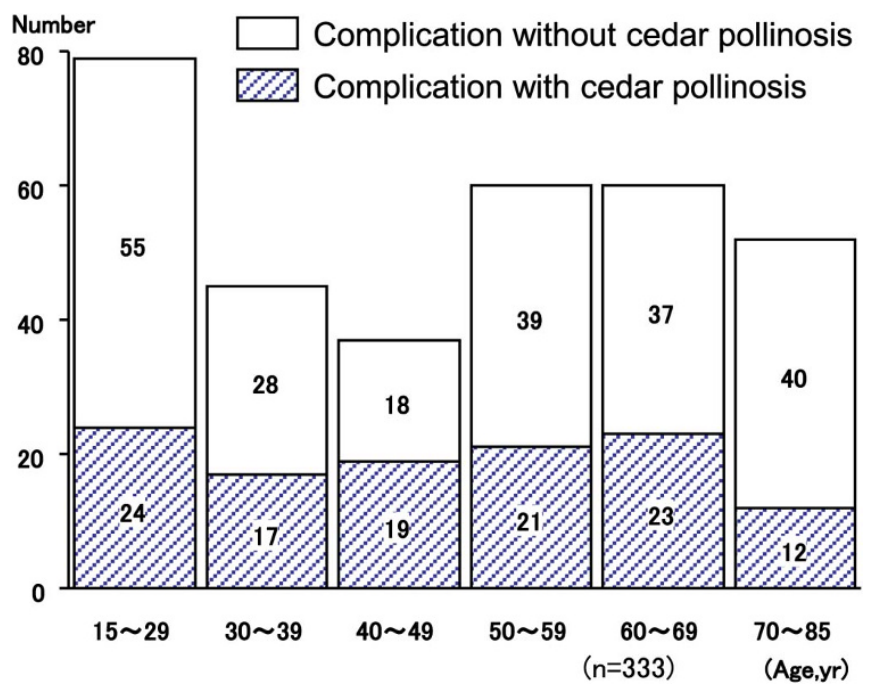

FIGURE 1. Distribution of the age of patients with asthma with or without complication with Japanese cedar pollinosis. Number of the patients was demonstrated. Adapted from Ueno et al (2002) with permission from Arerugi. ${ }^{6}$ during Japanese cedar pollen season. Among 41 patients whose asthma was well controlled, but was aggravated during the Japanese cedar pollen season, 13 patients showed a decrease in morning peak expiratory flow more than $10 \%$ during Japanese cedar pollen season compared with the baseline values (Fig. 2). However, it should be taken into account that the effect of virus infection, the most potent inducer of

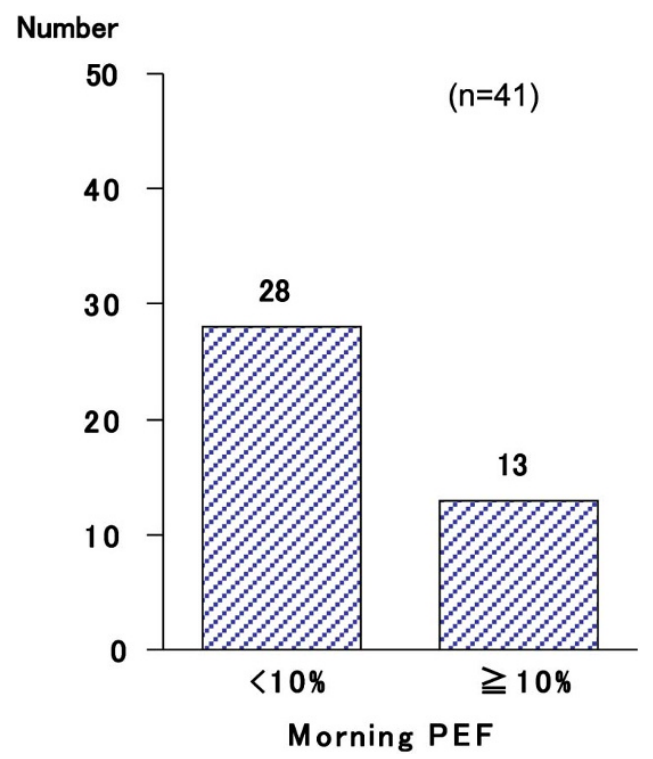

FIGURE 2. Number of patients whose morning peak expiratory flow decreased more or less than $10 \%$ compared with the baseline values was demonstrated. Adapted from Ueno et al (2002) with permission from Arerugi. ${ }^{6}$ 
asthma exacerbation, was not ruled out in this study. Next, we compared the patients' characteristics between the group that had and the group that did not have exacerbation of their asthma symptoms during Japanese cedar pollen season (Table 2). Among 116 asthmatic patients with Japanese cedar pollinosis, airway hyperresponsiveness was determined in 44 patients before the season of Japanese cedar pollen. Although the level of histamine- $\mathrm{PC}_{20}$ in the group in which asthmatic symptoms were exacerbated was lower than that in the group in which asthmatic symptoms were not exacerbated, they did not show statistical significance. Total IgE levels and specific $\mathrm{IgE}$ levels to Japanese cedar pollen showed no significant difference between the 2 groups.

According to the article reported by Fukushi et al, ${ }^{6}$ the prevalence of Japanese cedar pollinosis in 151 patients with asthma was $47.0 \%$, and $54.9 \%$ of the asthmatic patients with concomitant Japanese cedar pollinosis was associated with aggravation of asthma symptoms and reduced asthma control during Japanese cedar pollen season. These researchers also showed that the asthmatic patients with concomitant Japanese cedar pollinosis had lower levels of peak expiratory flow during the Japanese cedar pollen season.

These reports including ours suggest that the prevalence of Japanese cedar pollinosis in patients with adult asthma might be $30 \%$ to $50 \%$, and $30 \%$ to $60 \%$ of adult asthmatic patients with concomitant pollinosis might be associated with exacerbation of asthma during Japanese cedar pollen season. However, it is noteworthy that the diagnosis of Japanese cedar pollinosis is sometimes difficult, and the prevalence of Japanese cedar pollinosis has been changed decade by decade and by the area.

TABLE 2. Patient Characteristics on Exacerbation During Japanese Cedar Pollen Season

\begin{tabular}{|c|c|c|}
\hline & Exacerbation $(+)$ & Exacerbation (-) \\
\hline No. patients & 41 & 75 \\
\hline Age, yr & $49.5 \pm 2.9$ & $46.5 \pm 1.9$ \\
\hline \multicolumn{3}{|l|}{ Sex, n $(\%)$} \\
\hline Male & $16(39.0)$ & $30(40.0)$ \\
\hline Female & $25(61.0)$ & $45(60.0)$ \\
\hline Type Atopic, n (\%) & $31(75.6)$ & $51(68.0)$ \\
\hline Nonatopic & $10(24.4)$ & $24(32.0)$ \\
\hline Duration of asthma, yr & $16.8 \pm 2.2$ & $15.2 \pm 1.6$ \\
\hline IgE RIST (U/mL) & $532.2 \pm 101.1$ & $542.7 \pm 75.7$ \\
\hline Cedar RAST (UA/mL) & $25.1 \pm 5.5$ & $22.4 \pm 8.1$ \\
\hline Histamine- $\mathrm{PC}_{20}(\mu \mathrm{g} / \mathrm{mL})$ & $841 \pm 288(n=15)$ & $1375 \pm 886(\mathrm{n}=29)$ \\
\hline \multicolumn{3}{|l|}{ Severity of asthma, n (\%) } \\
\hline Mild & $6(14.6)$ & $21(28.0)$ \\
\hline Moderate & $22(53.7)$ & $39(52.0)$ \\
\hline Severe & $13(31.7)$ & $15(20.0)$ \\
\hline \multicolumn{3}{|l|}{ Therapy, n (\%) } \\
\hline BDP & $34(82.9)$ & $59(78.7)$ \\
\hline Slow-release theophylline & $37(90.2)$ & $57(76.0)$ \\
\hline Oral $\beta 2$-agonists & $21(51.2)$ & $28(37.3)$ \\
\hline Anti-allergic agents, n (\%) & $5(12.2)$ & $14(18.7)$ \\
\hline Oral steroids, n (\%) & $2(4.9)$ & $4(5.3)$ \\
\hline
\end{tabular}

Adapted from Ueno et al (2002) with permission from Arerugi. $^{6}$

\section{HYPOTHETIC MECHANISMS OF JAPANESE CEDAR POLIINOSIS-INDUCED ASTHMA EXACERBATIONS}

Grass and birch pollens are well known to induce asthma in Western countries. On the contrary, Japanese cedar pollen had not been thought to elicit asthma because the diameter of Japanese cedar pollen is approximately $30 \mu \mathrm{m}$ that is not thought to be able to reach to lower airways. However, Maeda et $\mathrm{al}^{7}$ reported that asthma can be provoked by Japanese cedar pollen. The asthmatic patients whose asthma symptoms were induced by Japanese cedar pollen developed the symptoms during the cedar pollen season. Among the 6 asthmatic patients whose asthma was induced by Japanese cedar pollen, 5 patients suffered from cedar pollinosis in addition to asthma and 1 patient had no pollinosis. In clinical practice, a number of patients with asthma are sensitized only to Japanese cedar pollen but not to other major antigens (mite, house dust, grass and birch pollens, fungus, and any pets). In these cases, patients usually get classified as nonatopic asthma. Instead, one should take into account that Japanese cedar pollen can cause asthma symptoms in these patients.

As shown above, Japanese cedar pollen is associated with exacerbations of asthma in a number of patients. Few reports have elucidated the mechanisms of how Japanese cedar pollen induces asthma exacerbations in patients with asthma with comorbid Japanese cedar pollinosis. On the contrary, there are many reports that elucidated how perennial $\mathrm{AR}$ is associated with exacerbation of asthma. ${ }^{8,9}$ Therefore, hypothetic mechanisms on how Japanese cedar pollen induces exacerbation in patients with asthma can be considered to be based on the mechanisms of AR-exacerbated asthma. Patients with asthma and concomitant pollinosis have a nasal obstruction during Japanese cedar pollen season, resulting in difficulty in nasal breathing. One of the most important nasal functions is trapping of environmental antigen, such as house dust and Japanese cedar pollen. Eventually, the absence of nasal trapping may facilitate the incursion of environmental antigen, resulting in the exacerbation of asthma. Moreover, insufficiency of humidity control and temperature control, resulting from nasal obstruction during Japanese cedar pollen season, is also considered to be associated with exacerbation of asthma. Furthermore, the direct impact of upregulated cytokines and chemokines from the upper airway to the lower airway and the systemic link also play a crucial role.

Both AR and asthma exhibit a similar pathophysiologic finding in upper and lower airways, respectively. They share similar findings of chronic inflammation with eosinophils, lymphocytes, macrophages, mast cells, cytokines, leukotrienes, and other inflammatory mediators ${ }^{10}$ (Fig. 3). It is thought that there may be more patients who potentially have comorbidity of AR and asthma than is reported previously because studies have demonstrated the presence of eosinophils in the upper airways of patients with asthma without nasal symptoms and the presence of eosinophils in the lower airways of patients with AR who do not exhibit bronchial hyperreactivity or other asthmatic symptoms. ${ }^{11,12}$ A potential mechanism linking AR and asthma is a systemic inflammatory response to allergen. Braunstahl et $\mathrm{al}^{13}$ reported that eosinophilic inflammation was 


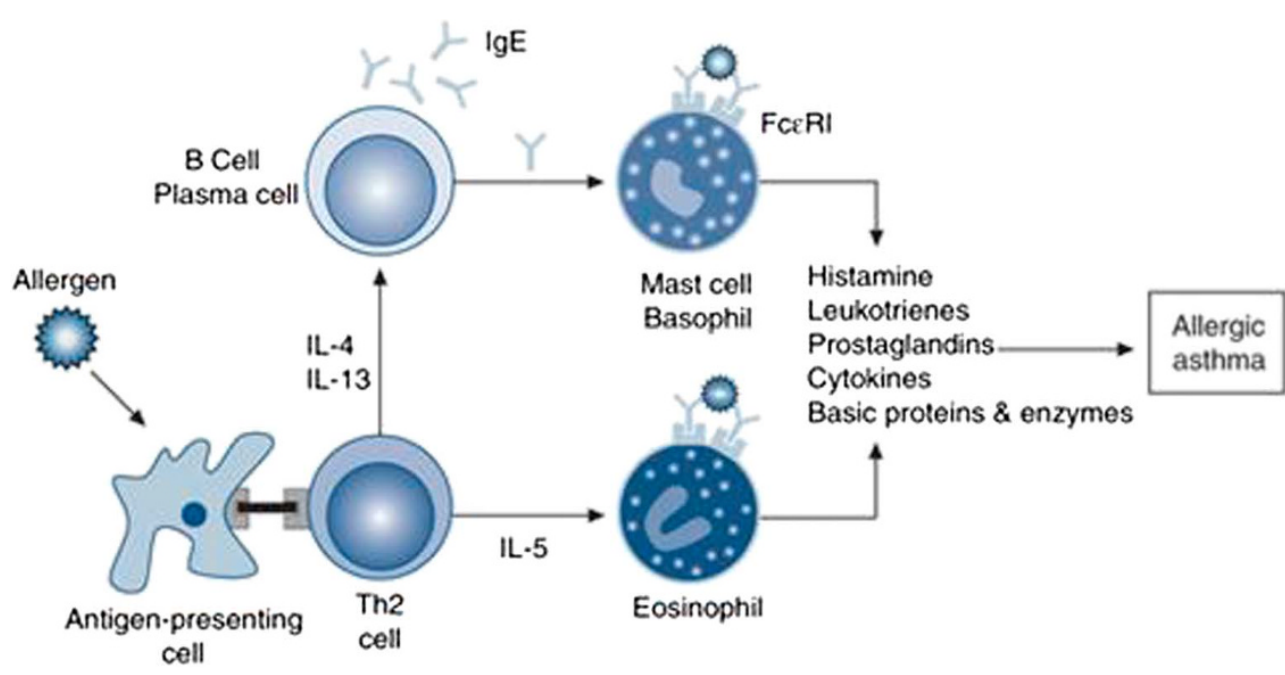

FIGURE 3. Mechanisms of allergic inflammation. Adapted from Holgate (2008) with permission from Clin Exp Allergy. ${ }^{11}$ increased in lower airways of patients with seasonal AR, 24 hours after nasal allergen challenge. Moreover, eosinophilic inflammation was enhanced by the segmental endobronchial allergen challenge with grass pollen in the nasal lamina propria and nasal epithelium of atopic subjects. ${ }^{14}$ These findings suggest a cross talk between allergic nasal inflammation and allergic lower airway inflammation, and the presence of more AR patients who have the potential of developing asthma and more asthmatic patients who have the potential of developing AR.

An early study suggested that a nasobronchial reflex arc would contribute to the linking of AR and asthma. ${ }^{15}$ Based on this hypothesis, nasal allergen challenge affects bronchial hyperresponsiveness through a reflex arc that involves trigeminal afferents and vagal efferents.

\section{TREATMENT STRATEGY OF ASTHMA WITH COMORBID AR OR JAPANESE CEDAR POLLINOSIS}

It is well known that the diagnosis of AR often precedes that of asthma. ${ }^{16}$ Rhinitis is an independent risk factor, intriguingly, not only in atopic individuals but also in nonatopic individuals for the subsequent development of asthma. ${ }^{17}$ Therefore, early intervention treatment of AR is hopefully considered as a preventive therapy for asthma. In fact, the Preventive Allergy Treatment study demonstrated that continuous pollen-specific immunotherapy for 3 years decreased the occurrence of asthma in patients with AR compared with that in patients with AR not receiving the immunotherapy. ${ }^{18}$ However, whether Japanese cedar pollinosis precedes asthma is still unknown.

The presence of concomitant $\mathrm{AR}$ is often associated with the difficulty of asthma control. ${ }^{19}$ In fact, here, we showed that AR increased the risk of asthma attacks and emergency room visit (Fig. 4). ${ }^{20}$ This leads to the question whether treating concomitant AR would produce better asthma-related outcomes in addition to the obvious benefits with regard to nasal symptoms. Studies have produced conflicting results regarding the effects of intranasal corticosteroids on the lower airways of patients with AR. Some of these studies ${ }^{21,22}$ have shown decreased bronchial hyperresponsiveness after treatment with intranasal corticosteroids, whereas other studies ${ }^{23,24}$ failed to show this. One study reported the positive effects of intranasal corticosteroids on the symptoms of asthma but not on bronchial responsiveness. ${ }^{25}$ The reason of this controversy has not been elucidated yet. However, at least, the differences of study designs and patient characteristics, including age and the concomitant presence or absence of asthma, are considered to be associated with this controversy. Despite the fact that intranasal steroids are the most effective agents for controlling symptoms of AR, second-generation antihistamines are still regarded as the first-line therapy for especially mild-tomoderate AR. Although antihistamines are not considered an effective treatment for asthma, Grant et $\mathrm{al}^{26}$ reported that an oral antihistamine given to patients with concomitant $\mathrm{AR}$ and asthma could improve persistent asthma symptoms during pollen season.

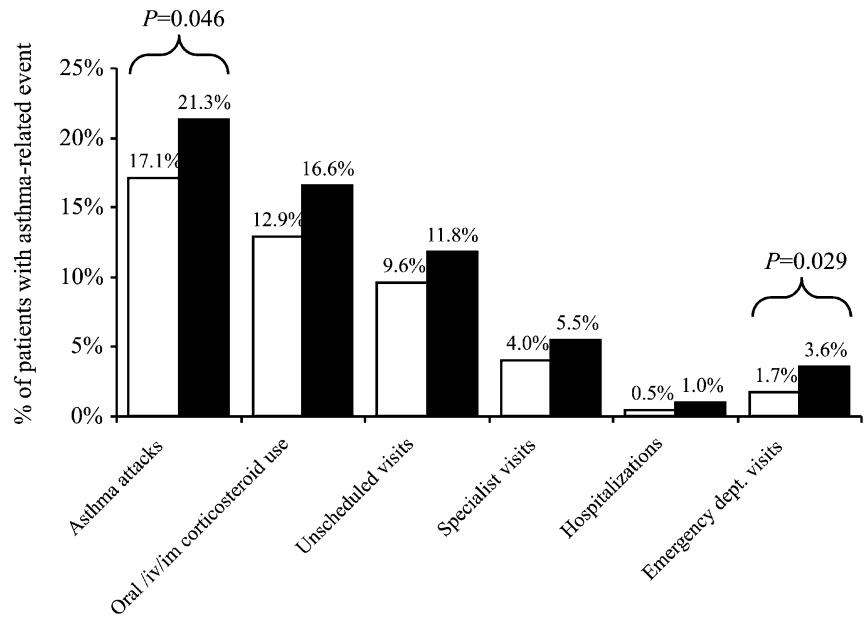

FIGURE 4. Asthma-related resource use and asthma attacks according to the presence of concomitant AR (\% patients). $\square$, asthma; $\mathbf{n}$, asthma with AR. Adapted from Bousquet et al (2005) with permission from Clin Exp Allergy. ${ }^{21}$ 
Leukotrienes are involved in the mechanisms of both asthma and AR. Therefore, leukotriene receptor antagonist (LTRA) is an effective drug for the patients with asthma and AR. In fact, COMPACT study (Clinical Outcomes with Montelukast as a Partner Agent to Corticosteroid Therapy) showed that LTRA was more effective in patients with asthma and AR than in those with asthma alone. ${ }^{27}$ Sagara et $\mathrm{al}^{28}$ demonstrated that a number of the patients with Japanese cedar pollinosis but not with asthma showed an increase in airway hyperresponsiveness during pollen season. They also demonstrated that this increase was inhibited by LTRA. Omalizumab, a humanized monoclonal anti-IgE antibody, is a novel drug for patients with severe asthma. The mechanism of omalizumab is inhibition of the binding of IgE to FceRI on mast cells and basophils. The effectiveness of omalizumab on AR and Japanese cedar pollinosis was demonstrated as well as on asthma. ${ }^{29,30}$ These data suggest that certain systemic drugs such as LTRAs and anti-IgE monoclonal antibody would produce better asthma-related outcomes in patients with asthma and concomitant AR.

\section{CONCLUSIONS}

There is a high prevalence of Japanese cedar pollinosis in patients with asthma. Concomitant Japanese cedar pollinosis is regarded as one of the factors leading to asthma exacerbations in patients with asthma and cedar pollinosis. Therefore, treatment of cedar pollinosis may contribute to better asthma control.

\section{REFERENCES}

1. Practical Guideline for the Management of Allergic Rhinitis in Japan: Perennial Phinitis and Pollinosis. 6th edn. Tokyo, Japan: Life Science; 2009.

2. Ozasa K, Dejima K, Takenaka H. Prevalence of Japanese cedar pollinosis among schoolchildren in Japan. Int Arch Allergy Immunol. 2002;128: 165-167.

3. Okamoto Y. Epidemiology of allergic rhinitis in Japan [in Japanese]. Igakuno Ayumi. 2008;216:329-333.

4. Leynaert B, Neukirch F, Demoly P, Bousquet J. Epidemiologic evidence for asthma and rhinitis comorbidity. J Allergy Clin Immunol. 2000;106(5 suppl): S201-S205.

5. Yamauchi K, Tamura G, Akasaka T, Chiba T, Honda K, et al. Analysis of the comorbidity of bronchial asthma and allergic rhinitis by questionnaire in 10,009 patients. Allergol Int. 2009; 58: 55-61.

6. Ueno K, Minoguchi K, Kohno Y, Oda N, Wada K, et al. Japanese cedar pollinosis is a risk factor for bronchial asthma in Japanese adult asthmatics [in Japanese]. Arerugi. 2002;51:565-570.

7. Fukushi M, Nagata M, Sakamoto Y. The effect of Japanese cedar pollinosis on patients with adult asthma [in Japanese]. Arerugi no Rinsho. 2003;23:64-65.

8. Maeda Y, Akiyama K, Shida T. A clinical study of Japanese cedar (Cryptomeria japonica) pollen-induced asthma. Allergol Int. 2008;57: 413-417.

9. Thomas M. Allergic rhinitis: evidence for impact on asthma. BMC Pulm Med. 2006;6(suppl 1):S4.

10. Meltzer EO, Szwarcberg J, Pill MW. Allergic rhinitis, asthma, and rhinosinusitis: diseases of the integrated airway. J Manag Care Pharm. 2004:10:310-317.

11. Holgate ST. Pathogenesis of asthma. Clin Exp Allergy. 2008;38:872-897.

12. Alvarez MJ, Olaguibel JM, García BE, Rodríquez A, Tabar AI, Urbiola E. Airway inflammation in asthma and perennial allergic rhinitis. Relationship with nonspecific bronchial responsiveness and maximal airway narrowing. Allergy. 2000;55:355-362.
13. Alvarez MJ, Olaguibel JM, Acero S, García BE, Tabar AI, Urbiola E. Effect of current exposure to Der $\mathrm{p} 1$ on asthma symptoms, airway inflammation, and bronchial hyperresponsiveness in mite-allergic asthmatics. Allergy. 2000;55:185-190.

14. Braunstahl GJ, Overbeek SE, Kleinjan A, Prins JB, Hoogsteden HC, Fokkens WJ. Nasal allergen provocation induces adhesion molecule expression and tissue eosinophilia in upper and lower airways. J Allergy Clin Immunol. 2001;107:469-476.

15. Braunstahl GJ, Kleinjan A, Overbeek SE, Prins JB, Hoogsteden HC, Fokkens WJ. Segmental bronchial provocation induces nasal inflammation in allergic rhinitis patients. Am J Respir Crit Care Med. 2000;161: 2051-2057.

16. Kaufman J, Chen JC, Wright GW. The effect of trigeminal resection on reflex bronchoconstriction after nasal and nasopharyngeal irritation in man. Am Rev Respir Dis. 1970;101:768-769.

17. Huovinen E, Kaprio J, Laitinen LA, Koskenvuo M. Incidence and prevalence of asthma among adult Finnish men and women of the Finnish Twin Cohort from 1975 to 1990 , and their relation to hay fever and chronic bronchitis. Chest. 1999;115:928-936.

18. Guerra S, Sherrill DL, Martinez FD, Barbee RA. Rhinitis as an independent risk factor for adult-onset asthma. J Allergy Clin Immunol. 2002; 109:419-425.

19. Möller C, Dreborg S, Ferdousi HA, Halken S, Høst A, et al. Pollen immunotherapy reduces the development of asthma in children with seasonal rhinoconjunctivitis (the PAT-study). J Allergy Clin Immunol. 2002;109:251-256.

20. Magnan A, Meunier JP, Saugnac C, Gasteau J, Neukirch F. Frequency and impact of allergic rhinitis in asthma patients in everyday general medical practice: a French observational cross-sectional study. Allergy. 2008;63:292-298.

21. Bousquet J, Gaugris S, Kocevar VS, Zhang Q, Yin DD, Polos PG, Bjermer L. Increased risk of asthma attacks and emergency visits among asthma patients with allergic rhinitis: a subgroup analysis of the investigation of montelukast as a partner agent for complementary therapy. Clin Exp Allergy. 2005;35:723-727.

22. Watson WT, Becker AB, Simons FE. Treatment of allergic rhinitis with intranasal corticosteroids in patients with mild asthma: effect on lower airway responsiveness. J Allergy Clin Immunol. 1993;91(1 pt 1):97-101.

23. Corren J, Manning BE, Thompson SF, Hennessy S, Strom BL. Rhinitis therapy and the prevention of hospital care for asthma: a case-control study. J Allergy Clin Immunol. 2004;113:415-419.

24. Thio BJ, Slingerland GL, Fredriks AM, Nagelkerke AF, Scheeren RA, et al. Influence of intranasal steroids during the grass pollen season on bronchial responsiveness in children and young adults with asthma and hay fever. Thorax. 2000;55:826-832.

25. Dahl R, Nielsen LP, Kips J, Foresi A, Cauwenberge P, et al; SPIRA Study Group. Intranasal and inhaled fluticasone propionate for polleninduced rhinitis and asthma. Allergy. 2005;60:875-881.

26. Stelmach R, do Patrocínio T, Nunes M, Ribeiro M, Cukier A. Effect of treating allergic rhinitis with corticosteroids in patients with mild-tomoderate persistent asthma. Chest. 2005;128:3140-3147.

27. Grant JA, Nicodemus CF, Findlay SR, Glovsky MM, Grossman J, et al. Cetirizine in patients with seasonal rhinitis and concomitant asthma: prospective, randomized, placebo-controlled trial. J Allergy Clin Immunol. 1995;95(5 pt 1):923-932.

28. Price DB, Hernandez D, Magyar P, Fiterman J, Beeh KM, et al; Clinical Outcomes with Montelukast as a Partner Agent to Corticosteroid Therapy (COMPACT) International Study Group. Randomised controlled trial of montelukast plus inhaled budesonide versus double dose inhaled budesonide in adult patients with asthma. Thorax. 2003;58:211-216.

29. Sagara H, Yukawa T, Kashima R, Okada T, Fukuda T. Effects of pranlukast hydrate on airway hyperresponsiveness in non-asthmatic patients with Japanese cedar pollinosis. Allergol Int. 2009;58:277-287.

30. Casale TB, Condemi J, LaForce C, Nayak A, Rowe M, et al; Omalizumab Seasonal Allergic Rhinitis Trail Group. Effect of omalizumab on symptoms of seasonal allergic rhinitis: a randomized controlled trial. JAMA. 2001;286:2956-2967.

31. Okubo K, Ogino S, Nagakura T, Ishikawa T. Omalizumab is effective and safe in the treatment of Japanese cedar pollen-induced seasonal allergic rhinitis. Allergol Int. 2006;55:379-386. 